\title{
A new approach to attenuate the effects of disturbances: A discrete linear systems
}

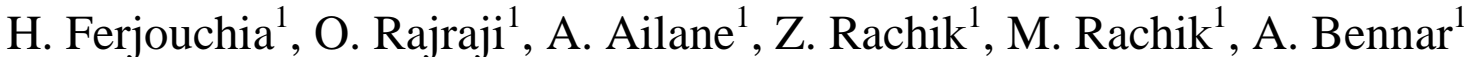 \\ ${ }^{I}$ M.I.T.I. Laboratory, faculty of sciences Ben M'sik, University Hassan II, mohammedia-Casablanca, Morocco
}

\begin{abstract}
In this article, we consider the perturbed controlled linear system described by the difference equation $x_{i+1}^{\omega}=A x_{i}^{\omega}+B u_{i}, x_{0}^{\omega}=x_{0}+\omega$ and the corresponding outputy ${ }_{i}^{\omega}=C x_{i}^{\omega}$, we suppose that $\omega$ is a disturbance which infects the system. Obliged to take into account the undesirable perturbation $\omega$, we investigate also in this work a feedback control $u_{i}=K x_{i}^{\omega}$ which allows to eliminate or to attenuate the effects of $\omega$. To illustrate the obtained results, various examples are presented.
\end{abstract}

Keywords: Discrete-time systems, perturbation analysis, stability, pole placement controllability, Ackermann's theorem, Milman's theorem, tolerance.

\section{Introduction}

During the modeling of a physical system, we cannot avoid some unwanted parameters that can have very negative effects on the evolution of the latter. Theses disturbances that are generally unavoidable could affect the different components of the system such as dynamic, control, observations, initial conditions ...

To solve this problem scientist came up with several approaches and techniques.

In [2] [10] [11] Rachik and al have proposed on approach which, instead of eliminating the effects of the disturbances, allows us to localize those whose effects don't go beyond the tolerance level that was fixed before. To follow this approach naturally, we consider in this paper the discrete disturbed linear system given by

and augmented by the output

$$
\left\{\begin{array}{c}
x_{i+1}^{\omega}=A x_{i}^{\omega}+B u_{i} \\
x_{0}^{\omega}=x_{0}+\omega
\end{array}\right.
$$

$$
y_{i}^{\omega}=C x_{i}^{\omega}
$$
objective

Our aim is to determine under certain conditions the control $u_{i}=K x_{i}^{\omega}$, which allows us to reach the

$$
\left\|y_{i}^{\omega}-\bar{y}_{l}\right\| \leq \epsilon, \quad \forall i \geq 0
$$

Where $\overline{y_{l}}$ is the no disturbed output, that is to say corresponding to $\omega=0$ and where $\varepsilon$ is a tolerance level fixed before. ie $u_{i}=C y_{i}^{\omega}$.

In the next part of this work, we deal the same problem, with using closed-loop controls the output $y_{i}^{\omega}$,

\section{Statement of the problem}

Let us consider the discrete perturbed system

$$
\left\{\begin{array}{c}
x_{i+1}^{\omega}=A x_{i}^{\omega}+B u_{i} \\
x_{0}^{\omega}=x_{0}+\omega
\end{array}, \quad \mathrm{i} \geq 0 \text { and } \forall \omega \in \Omega\right.
$$

where $A \in \mathcal{L}\left(\mathbb{R}^{n}\right), B \in \mathcal{L}\left(\mathbb{R}^{m}, \mathbb{R}^{n}\right)$, $\omega$ is unavoidable disturbance which affect the initial state $\mathrm{x}_{0}$, for realistic and technical raison, we suppose that $\omega \in \Omega$ where $\Omega \subset \mathbb{R}^{\mathrm{n}}$ is a convex open, bounded set with vertices $\operatorname{vert}(\Omega)=\left\{\omega_{1}, \omega_{2}, \cdots, \omega_{s}\right\}$ and with $0 \in \check{\Omega}(\stackrel{\Omega}{ }$ is the interior of $\Omega$ ).

The system's output is described by the difference equation (1) $y_{i}^{\omega}=C x_{i}^{\omega} i \geq 0 ; \quad C \in \mathcal{L}\left(\mathbb{R}^{n}, \mathbb{R}^{p}\right)$.

The control variable $u_{i}=K x_{i}^{\omega}$ where $K \in \mathcal{L}\left(\mathbb{R}^{n}, \mathbb{R}^{m}\right)$ is introduced to eliminate or to attenuate the effect of the perturbation $\omega$, that means

(2) $\left\|y_{i}^{\omega}-\bar{y}_{l}\right\| \leq \epsilon, \quad \forall i \geq 0$ and $\forall \omega \in \Omega$

where $\varepsilon>0$ is a threshold of tolerance and $\bar{y}_{l}$ the uninfected output, that's mean the output corresponding to $\omega=0,\|\cdot\|$ is the norm defined on $\mathbb{R}^{P}$ by

$$
\left(\left\|\begin{array}{c}
x_{1} \\
\vdots \\
x_{p}
\end{array}\right\|\right)=\max _{1 \leq i \leq n}\left|x_{i}\right|
$$




\section{Some technical results}

Let $K \in \mathcal{L}\left(\mathbb{R}^{n}, \mathbb{R}^{m}\right)$ and $u=\left(u_{i}\right)_{i \geq 0}$ the feedback control defined by $u_{i}=K x_{i}^{\omega}$, the corresponding output is which implies that

$$
y_{i}^{\omega}=C(A+B K)^{i}\left(x_{0}+\omega\right)
$$

consequently, the inequality (2) can be written

$$
y_{i}^{\omega}-\bar{y}_{l}=C(A+B K)^{i} \omega
$$

(3) $\left\|C(A+B K)^{i} \omega\right\| \leq \epsilon, \quad \forall \omega \in \Omega$ and $\mathrm{i} \geq 0$

it follows from (3) that the construction of a gain $K \in \mathcal{L}\left(\mathbb{R}^{n}, \mathbb{R}^{m}\right)$ solution of our problem, derived from an infinite number of inequality, to cope with this problem, we begin by establishing the following result

Proposition 3.1: there is equivalence between the following conditions

1. Forany $i \geq 0$ and for any $\omega \in \Omega$

2. For any $i \geq 0$ and for $\omega \in\left\{\omega_{1}, \omega_{2}, \cdots, \omega_{s}\right\}$

$$
\left\|C(A+B K)^{i} \omega\right\| \leq \epsilon
$$

$\left\|C(A+B K)^{i} \omega\right\| \leq \epsilon$

Proof. As the implication $(1 \Rightarrow 2)$ is obvious, so we must show the second one. Indeed, according to KreinMilman theorem [4] [5], for all $\omega \in \Omega$ there exist $\beta_{1} \geq 0, \cdots, \beta_{s} \geq 0$ such as $\sum_{i=1}^{s} \beta_{j}=1$ and $\omega=\beta_{1} \omega_{1}+\cdots+$ $\beta_{s} \omega_{s}$ then

$$
\begin{gathered}
\left\|C(A+B K)^{i} \omega\right\|=\left\|\sum_{j=1}^{s} \beta_{j} C(A+B K)^{i} \omega_{j}\right\| \\
\leq \sum_{j=1}^{s} \beta_{j}\left\|C(A+B K)^{i} \omega_{j}\right\| \\
\leq \sum_{j=1}^{s} \beta_{j} \epsilon=\epsilon \sum_{j=1}^{s} \beta_{j} \\
\leq \epsilon
\end{gathered}
$$

so we have

(4) $\left\|C(A+B K)^{i} \omega\right\| \leq \epsilon \quad \forall i \in \mathbb{N}, \forall \omega \in \Omega$

the proposition established allows to reduce the number of inequality (4) to small number, i.e.

(5) $\left\|C(A+B K)^{i} \omega\right\| \leq \epsilon \quad \forall i \in \mathbb{N}, \forall \omega \in\left\{\omega_{1}, \omega_{2}, \cdots \omega_{s}\right\}$

but, as we remark, the condition (5) generates an infinite number of constraints which don't allow the construction of gain $\mathrm{K}$. To overcome this difficulty, we assume that the spectrum of $\mathrm{A}+\mathrm{BK}$ is

$$
\sigma(A+B K)=\left\{\lambda_{1}, \lambda_{2}, \cdots \lambda_{n}\right\}
$$

we consider the basis $B=\left\{v_{1}, v_{2}, \cdots v_{s}\right\}$ of $\mathbb{R}^{\mathrm{n}}$ where $v_{i}$ are eigenvectors of $(\mathrm{A}+\mathrm{BK})$ associated to $\lambda_{i}$ and $\left\|v_{i}\right\|=1 . B_{c}=\left\{e_{1}, e_{2}, \cdots e_{s}\right\}$ is the canonical basis of $\mathbb{R}^{\mathrm{n}}$, so for all $\mathrm{x} \in \mathbb{R}^{\mathrm{n}}$, we have

whither the coordinates $\left(\begin{array}{c}x_{1} \\ \vdots \\ x_{n}\end{array}\right)$ and $\left(\begin{array}{c}z_{1} \\ \vdots \\ z_{n}\end{array}\right)$ are connected by relations

$$
x=\sum_{i=1}^{n} x_{i} e_{i} \text { and } x=\sum_{i+1}^{n} z_{i} v_{i}
$$

$$
\left(\begin{array}{c}
x_{1} \\
\vdots \\
x_{n}
\end{array}\right)=Q\left(\begin{array}{c}
z_{1} \\
\vdots \\
z_{n}
\end{array}\right)
$$

with $\left(q_{i j}\right)_{1 \leq i, j \leq n}$ is the transition matrix from the basis B to the basis $B_{c}$, furthermore

implying

$$
\begin{gathered}
(A+B K) x=(A+B K)\left[\sum_{i}^{n} z_{i} v_{i}\right] \\
=\sum_{i}^{n} z_{i}(A+B K) v_{i} \\
=\sum_{i}^{n} z_{i} \lambda_{i} v_{i}
\end{gathered}
$$


and we reason by recurrence, so it is easily shown that

$$
\begin{aligned}
(A+B K)^{2} x & =(A+B K)\left[\sum_{i+1}^{n} z_{i} \lambda_{i} v_{i}\right] \\
& =\sum_{i+1}^{n} z_{i} \lambda_{i}^{2} v_{i}
\end{aligned}
$$

$$
(A+B K)^{p} x=\sum_{i=1}^{n} z_{i} \lambda_{i}^{p} v_{i}, \forall p \in \mathbb{N}
$$

using (6), we have

$$
z_{i}=\sum_{i+1}^{n} q_{i j} x_{i}, \quad \forall i \in\{1, \cdots, n\}
$$

substituting this expression for (7), so

$$
(A+B K)^{p} x=\sum_{i=1}^{n}\left(\sum_{j=1}^{n} q_{i j} x_{j}\right) \lambda_{i}^{p} v_{i}
$$

implying

$$
\left\|(A+B K)^{p} x\right\| \leq \sum_{i=1}^{n}\left(\sum_{j=1}^{n}\left|q_{i j}\right|\left|x_{j}\right|\right)\left|\lambda_{i}\right|^{p}
$$

we note $\rho$ is the spectrum radious of $(\mathrm{A}+\mathrm{BK})$, then

and

$$
\rho=\sup _{\lambda_{i} \in \sigma(A+B K)}\left|\lambda_{i}\right| \text { and }\|x\|=\sup _{1 \leq j \leq n}\left\|x_{i}\right\|
$$

consequently

$$
\|Q\|=\sum_{i=1}^{n} \sum_{j=1}^{n}\left|q_{i j}\right|
$$

$$
\left\|(A+B K)^{p} x\right\| \leq\|Q\| \rho\|x\|
$$

\section{Solution of the problem}

Assuming the controllability of (A,B), using Ackermann's theorem, for given $\mathrm{n}$ real numbers

$$
\Gamma=\left\{0<\lambda_{1}<\lambda_{2} \cdots<\lambda_{n}<1\right\}
$$

there exists a matrix gain $K_{0} \in \mathcal{L}\left(\mathbb{R}^{n}, \mathbb{R}^{m}\right)$ such that $\sigma\left(A+B K_{0}\right)=\Gamma$ and so, according to inequality (8) (9) $\left\|(A+B K)^{p} x\right\| \leq\|Q\| \rho\|x\| \forall x \in \mathbb{R}^{\mathrm{n}}$

where we have the following proposition

Proposition 4.1: Given $\varepsilon>0$, if we assume that

$$
\|C\| \sup _{1 \leq i \leq s}\left\|\omega_{i}\right\| \leq \epsilon \text { and }\|C\|\|Q\| \sup _{1 \leq i \leq s}\left\|\omega_{i}\right\| \leq \epsilon
$$

so the control $u_{i}=K_{0} x_{i}^{\omega}$ allows $u$ s to reach the objective

$$
\left\|y_{i}^{\omega}-\bar{y}_{l}\right\| \leq \epsilon, \quad \forall i \geq 0 \text { and } \forall \omega \in \Omega
$$

Proof.According to proposition (3.1), it suffices that control $u_{i}=K_{0} x_{i}^{\omega}$ guarantees the following inequalities

$$
\left\|y_{i}^{\omega}-\bar{y}_{l}\right\| \leq \epsilon, \quad \forall i \geq 0 \text { and } \forall \omega \in\left\{\omega_{1}, \omega_{2}, \cdots \omega_{s}\right\}
$$

In fact, for $i=0$ and for $\omega_{r} \in\left\{\omega_{1}, \omega_{2}, \cdots \omega_{s}\right\}$ we have

$$
\begin{aligned}
&\left\|y_{0}^{\omega_{r}}-\overline{y_{0}}\right\|=\left\|C\left(x_{0}+\omega_{r}\right)-C x_{0}\right\| \\
& \leq\left\|C \omega_{r}\right\| \\
& \leq\|C\|\left\|\omega_{r}\right\| \\
& \leq\|C\| \sup \left\|\omega_{r}\right\| \leq \epsilon
\end{aligned}
$$

another for $\mathrm{i}>0$ and $\omega_{r} \in\left\{\omega_{1}, \omega_{2}, \cdots \omega_{s}\right\}$ we have

$$
\begin{gathered}
\left\|y_{i}^{\omega}-\bar{y}_{l}\right\|=\left\|C\left(A+B K_{0}\right)^{i} \omega_{r}\right\| \\
\leq\|C \quad\|\left\|\left(A+B K_{0}\right)^{i}\right\|\left\|\omega_{r}\right\| \\
\leq\|C\|\|Q\|\left\|\omega_{r}\right\| \\
\leq\|C\| \sup _{1 \leq r \leq s}\left\|\omega_{r}\right\| \leq \epsilon
\end{gathered}
$$

Example 4.1: Let's consider a perturbed discrete linear system described by

the output is supposed to be

$$
\left\{\begin{array}{l}
x_{i+1}^{\omega}=\left(\begin{array}{cc}
1 / 2 & 2 / 5 \\
-2 & 1
\end{array}\right) x_{i}^{\omega}+\left(\begin{array}{l}
0 \\
1
\end{array}\right) u_{i} \\
x_{0}^{\omega}=x_{0}+\omega, \quad \omega \in \Omega
\end{array}\right.
$$


where $\Omega$ is such that $\operatorname{vert}(\Omega)=\left\{\omega_{1}, \omega_{2}, \omega_{3}\right\}$ that means

$$
y_{i}^{\omega}=\left(\begin{array}{ll}
0 & 1 / 2
\end{array}\right) x_{i}^{\omega}
$$

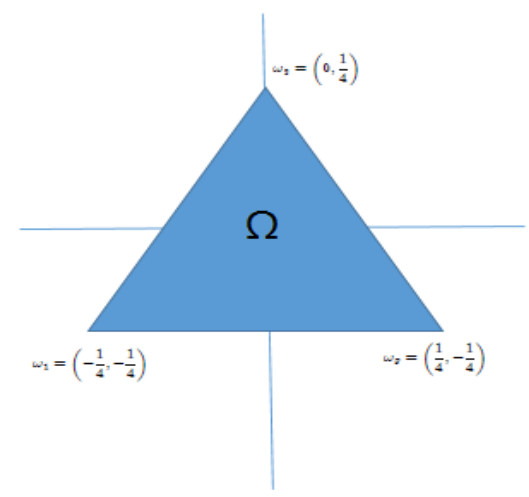

we have $\|C\|=1$ and $\sup \left\|\omega_{i}\right\|=1 / 4$, than other side

$$
\operatorname{rang}[B / A B]=2
$$

so, the system $(A, B)$ is controllable. Then and according to Ackermann's theorem [1], there exists a gain $\mathrm{K}_{0}$ that and

$$
\sigma(\mathrm{A}+\mathrm{BK})=\{0.2 ; 0.5\}
$$

$$
K=(2-4 / 5)
$$

and the other hand, the eigenvectors of the matrix $(\mathrm{A}+\mathrm{BK}) \operatorname{are} v_{1}=\left(\begin{array}{l}1 \\ 0\end{array}\right)$ and $v_{2}=\left(\begin{array}{c}1 \\ -3 / 4\end{array}\right)$, where $\left\|\mathrm{v}_{1}\right\|=\left\|\mathrm{v}_{2}\right\|=1$, so the transition matrix $\mathrm{Q}$ from $B=\left(v_{1} v_{2}\right)$ to $B_{c}=\left(e_{1}, e_{2}\right)$ is

Consequently, for $\varepsilon \geq 0.5$ we have

$$
Q=\left(\begin{array}{cc}
1 & 4 / 3 \\
0 & -4 / 3
\end{array}\right)
$$

$$
\|C\| \sup _{1 \leq i \leq 3}\left\|\omega_{i}\right\|=0.125 \leq \epsilon \text { and }\|C\|\|Q\| \sup _{1 \leq i \leq 3}\left\|\omega_{i}\right\|=0.46 \leq \epsilon
$$

and the control $u_{i}=K_{0} x_{i}^{\omega}$ allows to achieve the objective

$$
\left\|y_{i}^{\omega}-\bar{y}_{l}\right\| \leq \epsilon, \quad \forall i \geq 0 \text { and } \forall \omega \in \Omega
$$

Example 4.2: Let's consider the system

Augmented by the output

$$
\left\{\begin{array}{c}
x_{i+1}^{\omega}=\left(\begin{array}{cc}
-1 / 5 & -6 / 5 \\
11 / 5 & 3
\end{array}\right) x_{i}^{\omega}+\left(\begin{array}{c}
1 / 2 \\
-1
\end{array}\right) u_{i} \\
x_{0}^{\omega}=x_{0}+\omega, \quad \omega \in \Omega
\end{array}\right.
$$

The set $\Omega$ is $\operatorname{vert}(\Omega)=\left\{\omega_{1}, \omega_{2}, \omega_{3}, \omega_{4}\right\}$, i.e.,

$$
y_{i}^{\omega}=(-1 / 41 / 4) x_{i}^{\omega}
$$

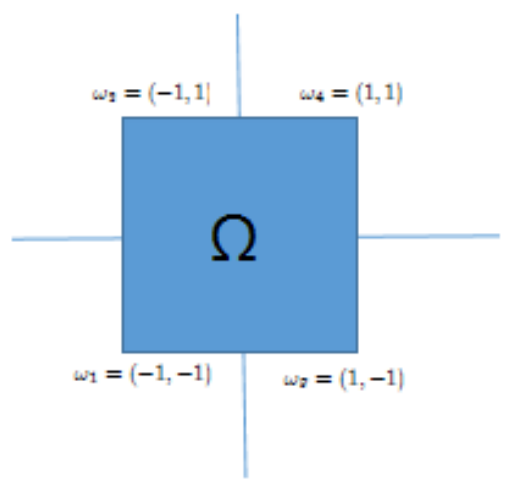


Therefore

$$
\begin{gathered}
\|C\|=1 / 4 \text { and } \sup _{1 \leq i \leq 4}\left\|\omega_{i}\right\|=1 \\
\operatorname{rang}[B / A B]=2
\end{gathered}
$$

Since the system $(A, B)$ is controllable. So according to Ackermann's theorem, we have

$$
\sigma(\mathrm{A}+\mathrm{BK})=\{0.6 ; 0.8\}
$$

and

$$
K=\left(\begin{array}{ll}
2 & 12 / 5
\end{array}\right)
$$

and the other hand, the eigenvectors of the matrix $(\mathrm{A}+\mathrm{BK})$ are $v_{1}=\left(\begin{array}{l}1 \\ 1\end{array}\right) a n d v_{2}=\left(\begin{array}{l}0 \\ 1\end{array}\right)$, where $\left\|\mathrm{v}_{1}\right\|=\left\|\mathrm{v}_{2}\right\|=1$, so the transition matrix $\mathrm{Q}$ from $B=\left(v_{1} v_{2}\right)$ to $B_{c}=\left(e_{1}, e_{2}\right)$ is

$$
Q=\left(\begin{array}{cc}
1 & 0 \\
-1 & 1
\end{array}\right)
$$

Consequently, for $\varepsilon \geq 0.8$, there exists a gain $\mathrm{K}_{0}$ such as $u_{i}=K_{0} x_{i}^{\omega}$ allows to reach the objective

$$
\left\|y_{i}^{\omega}-\bar{y}_{l}\right\| \leq \epsilon, \quad \forall i \geq 0 \text { and } \forall \omega \in \Omega
$$

\section{When the control is a feedback of the output}

In this section, we investigate a control $u_{i}=K x_{i}^{\omega}$ such that

$$
\left\|y_{i}^{\omega}-\bar{y}_{l}\right\| \leq \epsilon, \quad \forall i \geq 0 \text { and } \forall \omega \in \Omega
$$

which is equivalent to

$$
\left\|C(A+B K C)^{i} \omega\right\| \leq \epsilon, \quad \forall i \geq 0 \text { and } \forall \omega \in\left\{\omega_{1}, \omega_{2}, \cdots \omega_{s}\right\}
$$

assuming the controllability of $(\mathrm{A}, \mathrm{B})$ and using Ackermann's theorem [1], for

$$
\Gamma=\left\{0<\lambda_{1}<\lambda_{2} \cdots<\lambda_{n}<1\right\}
$$

there exists a gain $\mathrm{L} \in \mathcal{L}\left(\mathbb{R}^{\mathrm{n}}, \mathbb{R}^{m}\right)$ such that the spectre $\sigma(\mathrm{A}+\mathrm{BL})$ of $\mathrm{A}+\mathrm{BL}$ is

$$
\sigma(A+B L)=\Gamma
$$

if $\bar{B}=\left\{v_{1}, v_{2}, \cdots v_{n}\right\}$ are the associated eigenvectors, where \|\|$v_{i} \|=1$, as in section 4 , if $\mathrm{x} \in \mathbb{R}^{\mathrm{n}}$, we have

$$
x=\sum_{i=1}^{n} x_{i} e_{i} \text { and } x=\sum_{i}^{n} z_{i} v_{i}
$$

where $B_{c}=\left\{e_{1}, e_{2}, \cdots e_{s}\right\}$ is the canonical basis of $\mathbb{R}^{\mathrm{n}}$, we have

$$
\left\|(A+B L)^{r} x\right\| \leq \rho^{r}\|Q\|\|x\|
$$

where $\mathrm{Q}$ is the transition matrix from $\mathrm{B}$ to $B_{c}$, thus we have the following result (established in Section 4)

Proposition 5.1: Given $\varepsilon>0$ if we assume that

so the control $u_{i}=L x_{i}^{\omega}$ can achieves the objective

$$
\max \left(\|C\| \sup _{1 \leq i \leq n}\left\|\omega_{j}\right\|,\|C\|\|Q\| \sup _{1 \leq i \leq n}\left\|\omega_{i}\right\|\right) \leq \epsilon
$$

$$
\left\|y_{i}^{\omega}-\bar{y}_{l}\right\| \leq \epsilon, \quad \forall i \geq 0 \text { and } \forall \omega \in \Omega .
$$

Using this proposition, our problem is then to ensure, under certain conditions, the existence of $\mathrm{K}_{0} \in \mathcal{L}\left(\mathbb{R}^{\mathrm{n}}, \mathbb{R}^{m}\right)$ such as

(10) $L=\mathrm{K}_{0} C$

the following result solves the matricial equation (10)

Proposition 5.2: If we assume that $\operatorname{ker} C \subset$ kerLand $\operatorname{rang}(C)=p$, then the matrix $K_{0}=\left(L C^{\top}\right)\left(C C^{\top}\right)^{-1}$ is the unique solution of the equation (10).

Proof.Since $\operatorname{rang}(C)=p$, we have

i.e.

$$
\operatorname{ImC}=\mathbb{R}^{\mathrm{P}}
$$

which implies

$$
(\operatorname{Im} C)^{\perp}=\{0\}
$$

$$
\operatorname{ker}(C)^{\top}=\{0\}
$$

we have $\left\langle C C^{\top} x, x\right\rangle=\left|C^{\top} x\right|^{2} \geq 0$ and if $\left\langle C C^{\top} x, x\right\rangle=0$, then $\left|C^{\top} x\right|=0$ which implies $x \in \operatorname{ker} C^{\top}=\{0\}$ then $x=0$, consequently $C C^{\top}$ is positive definite, so it's invertible. On the other hand

$$
\mathbb{R}^{\mathrm{n}}=\operatorname{kerC} \oplus(\operatorname{ker} C)^{\top}
$$

moreover for any $\mathrm{x} \in \mathbb{R}^{\mathrm{n}}$ we have $x=x_{1}+x_{2}$ where $x_{1} \in \operatorname{kerC}$ and $x_{2} \in \operatorname{kerC}$. Since $(\operatorname{ker} C)^{\perp}=\operatorname{Im} C^{\top}$ where $x_{2}=C^{\top} z$ with $z \in \mathbb{R}^{p}$ so we have

$$
\begin{gathered}
K_{0} C x=L C^{\top}\left(C C^{\top}\right)^{-1} C\left[x_{1}+L C^{\top}\left(C C^{\top}\right)^{-1} C C^{\top} z\right] \\
=L C^{\top}\left(C C^{\top}\right)^{-1} C x_{1}+L C^{\top}\left(C C^{\top}\right)^{-1} C C^{\top} z \\
=L C^{\top} z
\end{gathered}
$$


$=L x_{1}+L x_{2}$

(because $x_{1} \in \operatorname{kerC} \subset$ kerL and so $L x_{1}=0$ ), consequently

i.e

$$
K_{0} C x=L x
$$

$$
K_{0} C=L
$$

to show that $\mathrm{K}_{0}$ is the unique solution of the equation (10), we assume that is another solution of (10), then

which implies

$$
\mathrm{HC}=\mathrm{L}
$$

that gives

$$
L C^{\top}=H C C^{\top}
$$

Consequently

$$
\left(L C^{\top}\right)\left(C C^{\top}\right)^{-1}=H
$$

Example 5.1: We consider the controlled system

$$
H=K_{0}
$$

$$
\left\{\begin{array}{c}
x_{i+1}^{\omega}=\left(\begin{array}{cc}
3 / 2 & -3 / 5 \\
-1 & 1
\end{array}\right) x_{i}^{\omega}+\left(\begin{array}{c}
-1 \\
1
\end{array}\right) u_{i} \\
x_{0}^{\omega}=x_{0}+\omega, \quad \omega \in \Omega
\end{array}\right.
$$

which is augmented by the output equation

$$
y_{i}^{\omega}=(1 / 2-1 / 4) x_{i}^{\omega}
$$

we have $\operatorname{vert}(\Omega)=\left\{\omega_{1}, \omega_{2}\right\}$ where $\Omega$ is such that

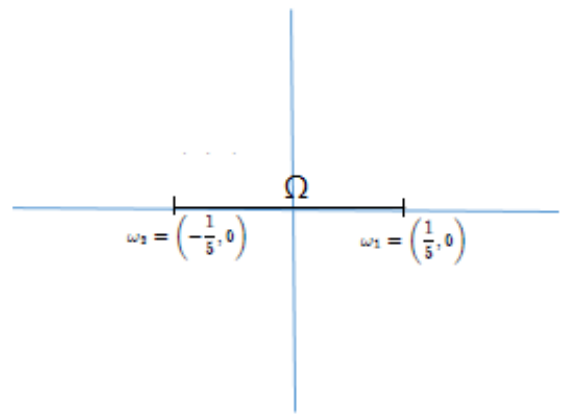

thus $\|C\|=1 / 2$ and $\sup _{1 \leq i \leq 2}\left\|\omega_{i}\right\|=1 / 5$, also we have

$$
\operatorname{rang}[B / A B]=2
$$

so the system $(\mathrm{A}, \mathrm{B})$ is controllable, and Ackermann's theorem ensures the existence of a matrix $\mathrm{L}$ that moreover

$$
\sigma(\mathrm{A}+\mathrm{BK})=\{0.3 ; 0.4\}
$$

The solution of the matricial equation $\mathrm{L}=\mathrm{KC}$ is

$$
L=(6 / 5-3 / 5)
$$

$$
K=12 / 5
$$

theeigenvectors of the matrix $(\mathrm{A}+\mathrm{BL})$ are $v_{1}=\left(\begin{array}{c}-1 / 2 \\ 1\end{array}\right)$ and $v_{2}=\left(\begin{array}{c}0 \\ 1\end{array}\right)$, where $\left\|\mathrm{v}_{1}\right\|=\left\|\mathrm{v}_{2}\right\|=1$, so the transition matrix $\mathrm{Q}$ from $B=\left(v_{1} v_{2}\right)$ to $B_{c}=\left(e_{1}, e_{2}\right)$ is

$$
Q=\left(\begin{array}{cc}
-2 & 0 \\
2 & 1
\end{array}\right)
$$

so, for $\varepsilon \geq 0.5$, the control $u_{i}=L x_{i}^{\omega}$ allows to reach the objective

$$
\left\|y_{i}^{\omega}-\bar{y}_{l}\right\| \leq \epsilon, \quad \forall i \geq 0 \text { and } \forall \omega \in \Omega \text {. }
$$

Example 5.2: We consider the system

augmented by

$$
\left\{\begin{array}{c}
x_{i+1}^{\omega}=\left(\begin{array}{cc}
-2 & 5 / 2 \\
6 / 5 & -3 / 5
\end{array}\right) x_{i}^{\omega}+\left(\begin{array}{c}
-1 \\
2 / 5
\end{array}\right) u_{i} \\
x_{0}^{\omega}=x_{0}+\omega, \quad \omega \in \Omega
\end{array}\right.
$$

$$
y_{i}^{\omega}=\left(1 / 3^{-1 / 3}\right) x_{i}^{\omega}
$$


$\operatorname{and} \operatorname{vert}(\Omega)=\left\{\omega_{1}, \omega_{2}, \omega_{3}\right\}$ where $\Omega$ is

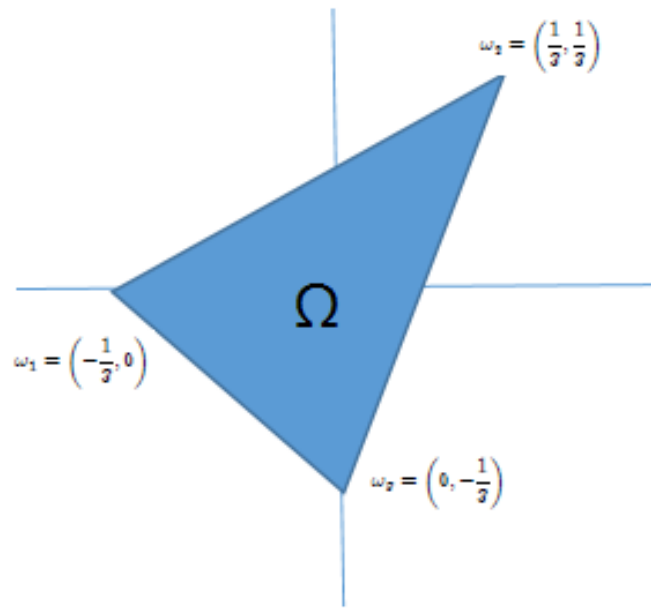

So $\|C\|=1 / 3$ and $\sup _{1 \leq i \leq 3}\left\|\omega_{i}\right\|=1 / 3$, Since the system (A,B) is controllable, So the ackermann's theorem ensures the existence of a matrix $\mathrm{L}$ that

moreover

$$
\sigma(\mathrm{A}+\mathrm{BK})=\{0.4 ; 0.5\}
$$

$$
L=(-5 / 25 / 2) \text {. }
$$

So the solution of the matricial equation $\mathrm{L}=\mathrm{KC}$ is

$$
K=5 / 2
$$

theeigenvectors of the matrix $(\mathrm{A}+\mathrm{BL})$ are $v_{1}=\left(\begin{array}{l}0 \\ 1\end{array}\right) a n d v_{2}=\left(\begin{array}{c}1 / 2 \\ 1\end{array}\right)$, and we have the transition matrix $\mathrm{Q}$ from $B=\left(v_{1} v_{2}\right)$ to $B_{c}=\left(e_{1}, e_{2}\right)$ is

$$
Q=\left(\begin{array}{cc}
-2 & 1 \\
2 & 0
\end{array}\right)
$$

so, for $\varepsilon \geq 0.6$, the control $u_{i}=L x_{i}^{\omega}$ allows to reach the objective

$$
\left\|y_{i}^{\omega}-\bar{y}_{l}\right\| \leq \epsilon, \quad \forall i \geq 0 \text { and } \forall \omega \in \Omega
$$

\section{Conclusion}

In this work, we considered a discrete linear system where the initial state is infected by disturbances $\omega$. A new approach to attenuate the effects of a disturbance was introduced. We investigate, in first part, the feedback control $u_{i}=K x_{i}^{\omega}$ to attenuate the effects of the disturbance, and in second one, we treated the same problem, with using closed-loop control the output $u_{i}=K y_{i}^{\omega}$. Numerical examples were provided to show the effectiveness.

\section{References}

[1] J. Ackermann, A. Bartlett, D. Kaesbauer, W. Sienel, and R. Steinhauser, Robust Control, Systems with Uncertain Physical Parameters. London, U.K.: Springer-Verlag, 1993

[2] A. Abdelhak, M. Rachik and E. Labriji. On the tolerable perturbed initial states: Discret systems. Journal of Applied Mathematical Sciences, 3(9), (2009) 429-442.

[3] M. Derouich and A. Boutayeb. Dengue Fever, A mathematical model with immunization program. In Handbook of Research on Systems Biology Applications in Medicine Daskalaki (eds) Medical Information Science Reference, (2009) 805-819.

[4] Jean-Baptiste Hiriart-Urruty, Claude Lemarechal, Convex Analysis and Minimization Algorithms I, Springer-Verlag, 1996

[5] DeaOjme, Sylvie Delabriere and Yves Raynaud. ConvexAnalysis-Universit 'e Pierre et Marie Curie - Paris 6, 2000/2001.

[6] L. Afifi, A. El Jai and M. Magri. Compensation problem in finite dimension linear dynamical systems. International Journal of ApplieMathematical Sciences, 2(45), (2008) 2219-2228

[7] Y. Qaraai, A. Bernoussi and A. El Jai. How to compensate a spreading disturbance for a class of nonlinear systems. Int. J. Appl. Math. Comput. Sci., 18(2), (2008) 1-17. Art. 213.

[8] L. Afifi, M. Bahadi and A. Chafiai. A regional asymptotic analysis of the compensation problem in distributed systems. International Journal of Applied Mathematical Sciences, 1(54), (2007) 2659-2686.

[9] A. Namir, F. Lahmidi, M. Laklalech and M. Azouazi. Numerical Approach for the Controllability of Distributed and Delay Systems. Journal of Applied Mathematical Sciences, 1(49), (2007) 2421-2442.

[10] M. Rachik, A. Tridane, M. Lhous, O. IdrissiKacemi and Z. Tridane. Maximal output admissible set and admissible perturbations set for nonlinear discrete systems. International Journal of Applied Mathematical Sciences, 1(32), (2007) 1581-1598.

[11] S. Saadi, Y. Rahhou, M. Rachik and O. El Kahlaoui. Observer design and admissible disturbances : A discrete disturbed system. International Journal of Applied Mathematical Sciences, 1(6), (2007) 241-266. 
[12] E. Zerrik and A. Kamal. Output controllability for semi linear distributed parabolic system. Journal of Dynamical and Control system, 13(2), (2007) 289-306.

[13] L. Afifi, A.El Jai and M. Merry, Detection and spy sensors in discrete distributed systems International Journal of Systems Sciences. 36, N12, 777-789, 2005

[14] L. Afifi, M. Bahadi, A. El Jai and A. El Mizane, The compensation problem in disturbed systems: Asymptotic analysis, approximations and numerical simulations International Journal of Pure and Applied Mathematics, 41, No 7, 927- 956, 2007.

[15] L. Afifi, M. Bahadi, A. Chafiai and A. El Mizane, Asymptotic compensation in discrete distributed systems: Analysis, approximations and simulation International Journal of Applied Mathematical Sciences , 2, N 3, 99-137, 2008.

[16] L. Afifi, M. Hakam, M. Bahadi and A. El Jai, Enlarged asymptotic compensation in discrete distributed systems NeuvimesJournesd'AnalyseNumriqueetd'Optimisation, JANO9. dcembre 2008 FST, Mohammedia. 